vol. 34 - $n^{\circ} 1 \mid 2018$

Mouvements migratoires d'hier et d'aujourd'hui en Italie

\title{
Les migrations italiennes : un aperçu statistique sur la longue durée
}

Italian Migration: A statistical overview on the long term

Migración italiana: una visión estadística a largo plazo

\section{Matteo Sanfilippo}

\section{OpenEdition}

Journals

\section{Édition électronique}

URL : https://journals.openedition.org/remi/9989

DOI : $10.4000 /$ remi.9989

ISSN : $1777-5418$

Éditeur

Université de Poitiers

Édition imprimée

Date de publication : 1 avril 2018

Pagination : 29-52

ISBN : 979-10-90426-61-0

ISSN : 0765-0752

Référence électronique

Matteo Sanfilippo, "Les migrations italiennes : un aperçu statistique sur la longue durée », Revue européenne des migrations internationales [En ligne], vol. 34 - $n^{\circ} 1$ | 2018, mis en ligne le 28 décembre 2019, consulté le 19 avril 2022. URL : http://journals.openedition.org/remi/9989 ; DOI : https://doi.org/ 10.4000/remi.9989 


\section{Les migrations italiennes: un aperçu statistique sur la longue durée}

\section{Matteo Sanfilippo ${ }^{1}$}

Entre 1818 et 1834, le journaliste et polygraphe Davide Bertolotti (Ponte, 1967) édite dans la revue I/ Raccoglitore (Le Collecteur), dont il est propriétaire et fondateur, des récits de voyage écrits par des voyageurs français et britanniques, ainsi que des œuvres, en italien, de voyageurs originaires de la Péninsule. En 1822, il publie Peregrinazioni (Pérégrinations), un ouvrage consacré aux voyages qu'il a effectués, mais aussi inventés dans les terres du Piémont et au-delà. Les deux premiers textes de ce volume relatent le voyage imaginaire à Buenos Aires de Vittorio, fils d'un hôtelier de Cadix en Espagne, et originaire d'un village du Piémont, situé au bord du lac d'Orta. L'auteur expose les raisons du départ de son personnage. Le récit de Bertolotti est très mélodramatique et mal structuré d'un point de vue littéraire. Toutefois, à travers cet ouvrage, l'auteur reconstruit l'émigration et notamment les rapports reliant Orta à Cadix et à Buenos Aires et présente le réseau migratoire qui s'est constitué entre I'Italie, l'Espagne et l'Amérique latine.

Selon Bertolotti (1822 : 3-50), au XVIIle siècle, les riverains des lacs situés entre le Piémont oriental et la Lombardie abandonnent leurs lieux de naissance, jugés très beaux, mais pas suffisamment riches. Les migrants cherchent du travail ailleurs et s'enrichissent souvent à Milan ou en Espagne, suivant une tradition qui remonte à la période durant laquelle le Duché de Milan était espagnol (1535-1714). Parmi ces migrants, nombreux sont ceux qui ouvrent des hôtels à Madrid, Barcelone et Cadix tout en conservant des liens avec leur lieu $\mathrm{d}$ 'origine. Après son mariage à Buenos Aires, Vittorio ainsi rentre à Cadix avec son épouse et se rend ensuite en Italie pour lui faire visiter son village paternel.

Dans la première partie de I'histoire, Bertolotti narre l'aventure de Vittorio : son père l'envoie étudier à Salamanque où il s'initie au commerce international, puis il part pour Buenos Aires. Il y exerce le commerce de peaux comme d'autres produits américains, suivant en cela les conseils de son père pour qui les révolutions latino-américaines sont en train d'ouvrir le trafic commercial avec l'Europe.

\footnotetext{
1 Historien, Professeur d'Histoire moderne à I'Université de Tuscia et Directeur de la Fondation Centro Studi Emigrazione, 4 rue S. Maria in Gradi, 01100 Viterbe, Italie; matteosanfilippo@unitus.it
} 
Avec Peregrinazioni, Bertolotti écrit une des premières "micro-histoires " ${ }^{2}$ sur la migration transnationale et transatlantique que nous connaissons. Ce récit s'accorde avec les documents conservés aux archives d'État de Turin (Archivio di Stato di Torino) et avec les analyses économiques (Gioia, 1804 : 29-32; Corti, 2000; Audenino, 2007). La documentation des archives turinoises confirme qu'entre le XVIIle et le XIXe siècle les Piémontais en Espagne sont employés dans I'hôtellerie et dans d'autres activités commerciales. En outre, comme le suggère Bertolotti, au début du XIXe siècle, les Italiens connaissent bien les réseaux migratoires commerciaux nationaux et internationaux, tant européens que transatlantiques. D'ailleurs, comme dans le cas de Vittorio, ceux que nous considérons aujourd'hui comme des réseaux nationaux à l'époque étaient internationaux; la région du lac d'Orta était reliée au royaume de Savoie, même avant d'en faire officiellement partie en 1817, tandis que, dès 1714, la région de Milan était intégrée dans l'empire austro-hongrois.

Les pages écrites par Bertolotti nous montrent en outre que, lorsque la Péninsule est partagée entre plusieurs puissances européennes, elle est traversée par des flux migratoires internationaux tant en entrée qu'en sortie. Ces courants migratoires relient entre eux les petits états italiens - communaux pendant le Bas Moyen Âge, régionaux à l'Époque moderne - ainsi que le territoire de la Péninsule aux royaumes continentaux et à leurs colonies transatlantiques. Ces flux au départ de l'Italie sont de nature hétérogène. Les exils politique et religieux, les guerres et les famines, mais aussi la tradition séculaire des métiers itinérants et marchands, les catastrophes climatiques et les fluctuations cycliques de l'économie italienne alimentent, à partir de du Bas Moyen Âge, les migrations à l'intérieur de la Péninsule, celles qui sont transalpines et, au début du XIXe siècle, les migrations transatlantiques (Pizzorusso, 2007).

Pour saisir la nature des flux migratoires enregistrés par la statistique italienne à partir des années $1870^{3}$, nous devons garder à l'esprit cette longue histoire migratoire. Après la création du royaume d'Italie en 1861, les modalités migratoires à l'intérieur et à l'extérieur du pays ne changent pas. Comme depuis le Bas Moyen Âge, les mobilités italiennes externes interagissent pendant la deuxième moitié du $\mathrm{XIX}^{\mathrm{e}}$ siècle avec les flux migratoires qui arrivent en Italie, se combinant entre eux ou bien alimentant la mobilité à l'intérieur de la Péninsule, notamment lorsqu'ils constituent une des étapes des migrations multiples (Corti et Sanfilippo, 2012; Sanfilippo, 2016).

\section{Une tradition migratoire d'Ancien régime}

Du XIVe au début du XIXe siècle, plusieurs régions italiennes ont engendré des migrations régulières et répétées qui ont perduré quasiment jusqu'à nos jours (Corti, 2003). II suffit de penser aux mouvements vers les vallées françaises et suisses depuis I'arc alpin (Albera et Corti, 2000) et à la mobilité agricole et pastorale du Sud de I'Italie centrale (Russo et Salvemini, 2007). Dans certains cantons, surtout le Nord de la Péninsule, ces déplacements ont été accompagnés

2 Pour le concept de "micro-histoire», voir Revel (1996).

3 Voir Statistica Generale del Regno d'Italia (1874 et 1884). Avant 1874, nous n'avons pas de données exception faite pour quelques années et pour quelques villes. 
par des pratiques séculaires (par exemple, le colportage) et ils ont transformés de manière significative la mentalité des individus et les conditions démographiques et économiques des familles. Dans les faits, celles-ci ont fini pour considérer la migration comme une stratégie habituelle et la seule manière pour s'assurer des ressources financières (Lorenzetti et Merzario, 2005). L'analyse approfondie de ces expériences met en évidence des constantes historiques qui demeurent inchangées depuis le Bas Moyen Âge par des pratiques migratoires trans-périodes qui s'achèvent avec la Première Guerre mondiale, mais qui depuis les zones de montagne, se prolongent jusqu'à nos jours (Viazzo, 2016).

Selon Pizzorusso (2001 et 2009), pour comprendre cette tradition migratoire, il faut prendre en compte quatre facteurs. En premier lieu, il est important d'étudier les périples des saisonniers depuis les montagnes vers les plaines italiennes et européennes. En deuxième lieu, il faut considérer les mouvements de la main-d'œuvre spécialisée se déplaçant à l'intérieur et à l'extérieur de la Péninsule, même si cette migration est parfois liée à des secteurs peu qualifiés du marché du travail. En troisième lieu, nous pouvons constater que la nécessité de migrer ne semble pas traumatiser ceux qui partent, même dans les cas les plus dramatiques liés à des raisons politique (comme dans le cas des expulsions opérées par les Communes au cours du Moyen Âge) ou religieuse (par exemple, les déplacements des vaudois, des protestants et des juifs). En quatrième lieu enfin, nous ne devons pas oublier que les départs et les retours s'appuient sur des réseaux bien établis et que, à quelques exceptions près, l'émigration italienne qui a eu lieu sous l'Ancien régime n'est pas un phénomène individuel. Bien au contraire, le départ est le plus souvent décidé au sein de la famille qui actionne les rapports de parenté et de voisinage et mobilise les ressources économiques disponibles. C'est précisément en raison de ce lien avec le cycle de vie familiale que chaque migrant prévoit un retour et que les plus âgés sont remplacés par les plus jeunes.

Ces dynamiques agissent jusqu'à la fin du XVIIIe siècle, quand certaines nouveautés voient le jour, dont notamment l'augmentation des migrations définitives; en effet, l'accroissement démographique de la Péninsule ne correspond pas à la croissance économique. Au début du XIXe siècle, au Nord comme au Sud de l'Italie, les migrations définitives remplacent les migrations temporaires et les migrations de longue durée remplacent les migrations saisonnières; les départs définitifs dépassent les retours et il est plus difficile de maintenir un équilibre entre les zones de départ et les zones d'arrivée. En outre, les itinéraires s'allongent et deviennent plus complexes. Des migrants partent en Russie ou aux Amériques par étapes géographiques et/ou générationnelles, comme le montre le cas de Vittorio et de son père : celui-ci arrive en Espagne et son fils part en Amérique latine. De nouveaux métiers se développent et certains travailleurs itinérants, qui auparavant s'adonnaient au colportage, voire à la mendicité, se spécialisent dans des secteurs qui deviennent des niches économiques liées aux migrations italiennes : le divertissement, la restauration et l'hôtellerie (Porcella, 2001).

\section{Les changements de la première moitié du XIXe siècle}

Une autre période politique qui va déterminer les changements des flux migratoires italiens internes et externes à la Péninsule se situe entre la Révolution française et l'occupation napoléonienne (Corsini, 1969 et 1993; Sanfilippo, 2008). 
En Italie, les différentes phases de la domination française génèrent un nombre important d'exilés politiques qui s'installent en France inaugurant ainsi la tradition de la politique du bannissement d'Italie qui se poursuit pendant tout le Risorgimento (durant de la seconde moitié du XIXe siècle). Cet exil politique a aussi pu se prolonger vers les Amériques, rejoignant les flux migratoires économiques et suivant leurs dynamiques (Isabella, 2014).

De même, pendant cette période, les préfets de Napoléon encouragent les travaux publics en Italie du Nord, ce qui attire les travailleurs d'autres régions et renverse alors un modèle migratoire qui traditionnellement avait incité les habitants du Nord de la Péninsule à se déplacer vers le Sud. Une fois les travaux terminés, cette main-d'œuvre se déverse dans les pays de I'Europe centrale et occidentale ou continue à circuler dans les régions du Nord de I'ltalie. À cette interaction entre migrations économique et politique, s'ajoute, avec la fin des guerres napoléoniennes, le fait que l'armée française en déroute se disperse provoquant des vagues migratoires touchant également I'Italie : ces anciens soldats cherchent un nouvel emploi et explorent plusieurs destinations migratoires, comme l'Afrique ou les pays transatlantiques.

Au milieu du XIXe siècle, les migrations transatlantiques acquièrent de plus en plus d'importance et entrainent l'essor des ports reliant I'Italie au continent américain. Vers 1850, la ville de Gênes représente le principal carrefour migratoire transatlantique et européen (on part de cette ville pour se rendre à Marseille ou en Angleterre) et assure les communications avec un arrière-pays très vaste incluant le triangle des Apennins (situé entre la Ligurie, l'Émilie et la Toscane), mais aussi les campagnes piémontaises et lombardes (Surdich, 2005). Toutefois, les Italiens ne partent pas seulement de Gênes; après y avoir travaillé pendant un certain temps, ils partent depuis d'autres pays européens (la France, la Belgique ou l'Angleterre) pour traverser I'océan Atlantique (de I'Espagne vers I'Amérique latine, de l'Angleterre vers les États-Unis ou le Canada). Avant I'unité politique de l'Italie, des travailleurs spécialisés dans des secteurs variés parcourent ainsi I'Europe et du pays dans lequel ils résident, se rendent ensuite aux Amériques (Porcella, 2001). Au milieu du XIXe siècle, les migrants de Biella (Piémont) se rendent d'abord en France et, de là, en Espagne, puis ensuite se dirigent vers les pays du Nouveau Monde (comme le montre le parcours du père de Vittorio dans le texte de Bertolotti) sans omettre d'également explorer la possibilité d'aller travailler aux États-Unis (Castronovo, 1986-1997). Un autre exemple encore, depuis le royaume des Deux-Siciles, des musiciens, des conteurs et des jongleurs circulent dans toute l'Europe, de Moscou à Madrid, pour ensuite traverser I'Océan (Cappelli, 2015). Nous ignorons les modalités exactes de ces déplacements ainsi que les raisons précises qui ont poussé des milliers des personnes à franchir l'Atlantique, mais il faut garder à l'esprit qu'à partir de la première moitié du XIXe siècle les journaux et les magazines propagent le mythe du continent américain comme terre d'avenir et de richesse (Rosoli, 1990; Albonico et Rosoli, 1994). Nombreuses sont alors les personnes qui commencent à préférer les terres lointaines des Amériques, dans l'espoir d'obtenir un succès improbable sur le Vieux Continent (Sanfilippo, 2008) et, dès la moitié du XIXe siècle, les pays des destinations transatlantiques entrent dans la tradition migratoire italienne en attirant des personnes qui exercent des métiers (surtout spécialisés) et en provenance de régions septentrionales (notamment la Lombardie et le Piémont) (Audenino, 1990). 
La relation entre les flux de nature politique et de nature économique devient plus évidente pendant le Risorgimento : la dimension de l'exil est fondamentale non seulement pour comprendre l'essor des émeutes et reconstruire les trajectoires des Italiens expatriés qui suivent les routes ouvertes par Giuseppe Mazzini et Giuseppe Garibaldi ${ }^{4}$ (Sanfilippo, 2009). Les anciens détenus qui ont participé aux émeutes contre l'Autriche ont inauguré l'ère des migrations politiques de masse, notamment ceux qui sont allés dans la prison du Spielberg; ils ont été très rapidement suivis par les révolutionnaires de 1848 en fuite. En général, ils optent pour l'Europe (Paris, Bruxelles et Londres); toutefois, ils ne négligent pas les Amériques, surtout Buenos Aires et New York, où ils fondent une presse en langue italienne qui devient un élément unificateur des communautés italophones, contribuant ainsi à I'essor du processus nationaliste italien (Franzina, 1995; Sergi, 2010).

\section{L'État-nation}

Dans les années qui suivent l'unité de l'Italie, les problèmes économiques de nombreuses régions italiennes confortent ces mécanismes migratoires (Sori, 1979; Fauri, 2015). La migration massive de la fin du XIXe siècle est le point culminant d'un processus commencé depuis longtemps qui garde certaines caractéristiques du passé, notamment la tendance à émigrer, réémigrer, rentrer. Le départ est en effet une réponse à la nouvelle conjoncture économique, mais aussi une façon de l'anticiper : comme le souligne Grossutti (2009), la véritable cause des migrations a été la peur de la misère et non la misère elle-même, surtout dans le Nord-Est de la Péninsule. De plus, il faut considérer que la nation italienne s'est constituée en absorbant progressivement des régions italophones. Le royaume d'Italie est proclamé en 1861, mais ses frontières s'élargissent en 1866, 1870 et 1918 , intégrant des régions où l'on parle italien, mais qui ont été façonnées par les dispositifs migratoires de l'empire d'Autriche (Franzina, 1976 et 2001).

Les premières statistiques des Italiens vivant à l'étranger ne sont donc pas complètement fiables, notamment parce que ces chiffres considèrent comme migrants ceux qui sont partis avant de devenir Italiens. En tout cas, ceux-ci ont donné vie à des communautés italophones sur lesquelles se sont appuyés ceux qui sont partis après 1861, 1866 et 1870 et qui, comme le montrent les données du tableau 1, ont élargi les pays de destination : en 1871, les Italiens se dirigent surtout vers l'Europe ; en 1881 les Amériques sont leur lieu de destination privilégié.

Tableau 1 : Effectifs des communautés italiennes par continent en 1871 et 1881

\begin{tabular}{lccccc}
\hline Année & Europe & Afrique & Amériques & Asie & Océanie \\
\hline 1871 & 155278 & 23645 & 87026 & 4016 & 740 \\
1881 & 373484 & 61003 & 381973 & 5625 & 2877 \\
\hline
\end{tabular}

Source : Statistica Generale del Regno d'Italia (1874 et 1884).

Dans le dernier quart du XIXe siècle, la main-d'œuvre excédentaire de la plaine du Pô migre en France et en Belgique ou, plus rarement, en Suisse et en Allemagne.

4 Giuseppe Mazzini (1805-1872) est un révolutionnaire et patriote italien républicain combattant pour la réalisation de l'unité italienne. Il est considéré avec Giuseppe Garibaldi (1807-1882), général, homme politique et patriote italien, comme I'un des " pères de la patrie " italienne. 
Ces migrants cherchent à rassembler le plus rapidement possible un capital pour acheter des terres dans leur région d'origine. Au contraire, les paysans de la Vénétie (région devenue italienne en 1866), du Frioul (région devenue en partie italienne en 1866), du Trentin et du Haut-Adige (région devenue italienne après la Première Guerre mondiale) migrent vers l'Amérique latine, où ils cherchent et trouvent des terres et ne souhaitent plus en partir, étant donné que leurs régions de départ se sont appauvries après la séparation de l'empire d'Autriche (Franzina, 2014). Les petits propriétaires terriens de I'Italie du Sud, évincés du marché ou acculés par les impôts, se dirigent vers les deux Amériques; ils seront imités par ceux qui partent depuis I'Italie centrale. Dans le but de rentrer un jour au pays, ces émigrés mettent en place des stratégies multiples, de la vente de leurs terrains avec possibilité de rachat, à l'aide économique de toute la famille pour envoyer un parent en éclaireur (De Clementi, 1999). Parallèlement, les anciens courants migratoires professionnels persistent et se consolident, notamment parmi les ouvriers spécialisés de I'Italie du Nord (Audenino, 1990; Corti, 1990). Au cours de cette même période, l'exil acquiert une nouvelle dimension destinée à se prolonger au $\mathrm{XXe}$ siècle : après les bannis des communes médiévales ou des états régionaux de l'époque moderne, après les exilés du Risorgimento, ce sont les membres des nouveaux mouvements sociaux qui partent. Les personnes qui avaient perdu la bataille pour la lutte des terres ${ }^{5}$ de la fin du XIXe siècle sont accompagnées par les socialistes, les anarchistes et, après 1921, par les communistes; ils forment des poches de résistance politique et sociale à l'étranger. Dans certains cas, la motivation politique renforce la motivation économique et les personnes suivent les mêmes routes migratoires; dans d'autres cas, ce sont des individus seuls, mais qui empruntent des routes désormais pluriséculaires (Degl'Innocenti, 1992).

Au cours des quinze premières années du XXe siècle, nous assistons à une augmentation du nombre de départs, mais la Première Guerre mondiale impose un arrêt, au moins partiellement et, surtout, elle provoque le retour précipité de nombreux émigrés et de leurs enfants, qui étaient nés hors les frontières nationales, mais qui avaient baignés dans la rhétorique du Risorgimento (Prezioso, 2010; Gastaut et Kronenberger, 2014; Franzina, 2015 et 2017a). Le nombre élevé de retours qui accompagne le déclenchement de la guerre est dû à la crainte de ne plus pouvoir rentrer à cause du conflit; toutefois, nous ne devons pas sous-évaluer l'effet de l'enrôlement dans l'armée pour défendre la patrie. En tout cas, la guerre n'interrompt pas les déplacements vers les pays européens, même rivaux, tant est si bien que les Italiens continuent à chercher du travail sur les territoires autrichiens et allemands (Salvetti, 1998). En outre, I'avancée de l'armée autrichienne provoque le déplacement des Vénitiens et Frioulans vers Milan et Turin : ces flux migratoires internes se sont prolongés jusqu'aux années 1960 (Ermacora, 2013 ; Franzina, 2017b).

La paix est suivie par la reprise immédiate de l'exode vers l'étranger; toutefois, à la fin des années 1910, le climat international n'est plus favorable aux migrations. Les empires de l'Europe centrale, destination traditionnelle des migrants vénitiens et frioulans, sont morcelés et vivent une grave crise économique; dans la même période, les états américains, qui ont participé à la guerre, ferment leurs frontières. 1918 marque en même temps la fin de la guerre et la fin d'un processus migratoire de très longue durée et qui s'est prolongé jusqu'au début du XXe siècle (Franzina, 1982).

5 Dans le dernier quart du siècle, au sud comme au nord-est du pays les paysans, appauvris, se soulèvent contre les propriétaires fonciers et l'Etat (Cazzola, 1983; Renda, 1977). 
Tableau 2 : Flux annuels d'émigrants italiens selon le continent de destination (1876-1918)

\begin{tabular}{|c|c|c|c|c|c|c|}
\hline Année & Europe & Afrique & Amériques & Asie & Océanie & Total \\
\hline 1876 & 86617 & 2544 & 19610 & & & 108771 \\
\hline 1877 & 76515 & 1313 & 21169 & & 216 & 99213 \\
\hline 1878 & 72367 & 2944 & 20743 & 20 & 194 & 96268 \\
\hline 1879 & 80004 & 2679 & 37075 & 42 & 31 & 119831 \\
\hline 1880 & 84224 & 2555 & 33080 & 27 & 15 & 119901 \\
\hline 1881 & 92107 & 2792 & 40871 & 52 & 10 & 135832 \\
\hline 1882 & 93930 & 7855 & 59695 & 53 & 29 & 161562 \\
\hline 1883 & 98665 & 6835 & 63388 & 49 & 164 & 169101 \\
\hline 1884 & 87558 & 3754 & 55467 & 65 & 173 & 147017 \\
\hline 1885 & 78232 & 6217 & 72490 & 96 & 158 & 157193 \\
\hline 1886 & 80406 & 4964 & 82166 & 62 & 231 & 167829 \\
\hline 1887 & 82474 & 3451 & 129463 & 61 & 216 & 215665 \\
\hline 1888 & 82941 & 3334 & 204264 & 34 & 163 & 290736 \\
\hline 1889 & 92631 & 2413 & 123181 & 31 & 156 & 218412 \\
\hline 1890 & 100259 & 2228 & 113027 & 49 & 291 & 215854 \\
\hline 1891 & 103885 & 2401 & 186472 & 89 & 784 & 293631 \\
\hline 1892 & 107025 & 2547 & 113807 & 144 & 144 & 223667 \\
\hline 1893 & 104482 & 3649 & 138299 & 267 & 54 & 246751 \\
\hline 1894 & 110757 & 2663 & 111459 & 376 & 68 & 225323 \\
\hline 1895 & 105273 & 3432 & 183919 & 403 & 154 & 293181 \\
\hline 1896 & 109928 & 3934 & 192998 & 155 & 467 & 307482 \\
\hline 1897 & 125310 & 2726 & 171294 & 89 & 436 & 299855 \\
\hline 1898 & 144528 & 3551 & 135193 & 72 & 371 & 283715 \\
\hline 1899 & 162899 & 4848 & 139934 & 231 & 427 & 308339 \\
\hline 1900 & 181047 & 5417 & 165627 & 156 & 535 & 352782 \\
\hline 1901 & 244298 & 9499 & 278176 & 382 & 890 & 533245 \\
\hline 1902 & 236066 & 11771 & 282586 & 319 & 767 & 531509 \\
\hline 1903 & 215943 & 10691 & 280413 & 540 & 389 & 507976 \\
\hline 1904 & 203942 & 16598 & 249574 & 376 & 701 & 471191 \\
\hline 1905 & 266982 & 13072 & 444724 & 788 & 765 & 726331 \\
\hline 1906 & 264883 & 11569 & 509348 & 1362 & 815 & 787977 \\
\hline 1907 & 276420 & 12685 & 414303 & 603 & 664 & 704675 \\
\hline 1908 & 240953 & 7351 & 237405 & 327 & 638 & 486674 \\
\hline 1909 & 219623 & 7098 & 397666 & 420 & 830 & 625637 \\
\hline 1910 & 242381 & 6670 & 400852 & 493 & 1079 & 651475 \\
\hline 1911 & 263966 & 7393 & 260372 & 505 & 1608 & 533844 \\
\hline 1912 & 294371 & 15725 & 399713 & 423 & 1214 & 711446 \\
\hline 1913 & 307627 & 6541 & 556325 & 423 & 1682 & 872598 \\
\hline 1914 & 241478 & 4951 & 230765 & 1397 & 561 & 479152 \\
\hline 1915 & 74389 & 5306 & 65877 & 100 & 347 & 146019 \\
\hline 1916 & 65209 & 3117 & 73832 & 39 & 167 & 142364 \\
\hline 1917 & 31439 & 2086 & 12940 & 17 & 14 & 46496 \\
\hline 1918 & 22986 & 1309 & 3985 & 27 & 4 & 28311 \\
\hline
\end{tabular}

Source : L. Prencipe (Prencipe et Sanfilippo, 2009).

6 Sauf mention contraire, tous les tableaux de l'article proviennent de Prencipe et Sanfilippo (2009). 
En 1918, le nombre des départs se réduit, surtout si l'on compare aux années précédentes, qui étaient pourtant des années de guerre : en 1917, on enregistre 46 496, en 1918 ils ne sont que 28311. En 1906 sont comptabilisés 787977 départs, dont 509348 pour les Amériques; en 1918, les départs pour les Amériques ne sont que 3985. Au cours de la même année, 22986 Italiens se rendent dans des pays européens, ce qui représente à peu près un dixième de la moyenne annuelle des années précédant la Grande Guerre.

\section{Le XXe siècle}

Si la période 1876-1918 marque la fin de pratiques migratoires datant de l'Ancien régime, les décennies suivantes sont celles de l'émergence d'un processus nouveau : les migrations, surtout internes, changent le visage de la Péninsule. Les Italiens partent des régions rurales ou des montagnes du Nord-Est du pays pour se rendre à Milan, Turin et Gênes; de I'Italie du Sud vers Rome, Naples et les villes du Nord, renforçant ainsi les mouvements de populations qui avaient débuté au moment de l'unité nationale (Trevès, 1976; Gallo, 2012; Ermacora, 2013). D'autres flux migratoires se mettent en place depuis plusieurs régions du Sud et du Nord-Est en direction de pays européens (comme la France) ou américains (notamment les États-Unis et l'Argentine). Dans ce dernier cas, il ne s'agit pas de nouvelles migrations, mais d'une conséquence du fait que les anciens migrants ne rentrent plus craignant de ne plus pouvoir repartir vers les Amériques. Ils interrompent ainsi la forme de migration circulaire entre lieux de départ et d'arrivée (Audenino et Tirabassi, 2008), c'est-à-dire le fait que la plupart des migrants rentraient après quelques années avant de repartir à nouveau. Par ailleurs, il faut aussi considérer la fuite de ceux qui s'opposent au régime fasciste, ainsi que de ceux qui n'étaient pas ouvertement des militants antifascistes, et qui optent notamment pour la France, à leurs yeux plus riche et surtout plus démocratique (Milza, 1986; Vial, 2007).

\section{L'entre-deux-guerres}

La fermeture des frontières migratoires américaines ralentit les déplacements des membres de la diaspora italienne. La principale loi restrictive en matière d'immigration est votée en 1924 aux États-Unis. Cet exemple est suivi par d'autres pays américains (Franzina, 1982), mais c'est surtout la grande crise économique de 1929 qui frappe violemment l'Amérique du Nord qui va mettre un terme à l'arrivée d'Italiens (Luconi et Pretelli, 2008). Entre temps, le Parti national fasciste une fois arrivé au pouvoir, cherche à diriger les mouvements de population vers les colonies et à canaliser les migrations internes. L'assainissement des marais pontins (Latium) et sardes, ainsi que la migration planifiée de familles provenant des marches et de la Vénétie dans ces régions ont eu comme conséquence les déplacements et la création de nouvelles agglomérations urbaines, comme les centres de Latina (Latium) et de Carbonia (Sardaigne) (Gallo, 2015). La mobilité interne à la Péninsule change de nature; les flux campagne-campagne ou bien campagne-petites villes du XIXe siècle (Nani, 2016) se transforment et les migrations alimentent le processus d'urbanisation. 
De même, la tendance toujours plus forte des migrants à s'installer en ville marque une rupture définitive avec la tradition migratoire et elle anticipe ce qui se concrétise après le second conflit mondial, à savoir le dépeuplement des montagnes, des collines et des plaines plus périphériques (Birindelli et al., 1978). Ce phénomène continue jusqu'au XXle siècle et il marque les transformations démographiques et géographiques italiennes (SVIMEZ, 2001-2016). De plus, la stabilisation de plusieurs communautés à l'étranger - par exemple aux ÉtatsUnis, mais aussi en France - contribue à l'essor des "Petites Italies " qui constituent un pôle d'attraction important pour les nouvelles vagues migratoires entre "I'Ancien et le Nouveau Monde "(Baldoli, 2004; Blanc-Chaléard et al., 2007; Mourlane et Païni, 2017).

Dans ce processus de reconfiguration, le Ventennio fasciste est une époque charnière, injustement ignorée pendant longtemps par de nombreux chercheurs travaillant sur les migrations. Aux affirmations et aux initiatives officielles contre les départs pour l'étranger et l'urbanisation correspond une réalité tout à fait différente. Les autorités mettent en place une rhétorique qui affirme que l'émigration est une "honte nationale" qu'il faut évincer : elle est continuellement condamnée et le gouvernement proclame qu'il faut "récupérer" les Italiens qui sont à l'étranger en leur offrant la possibilité de rentrer (Baldoli, 2003 ; Pretelli; 2010; Capece, 2015). Toutefois, cette propagande politique n'a que peu d'effet auprès des émigrés (Franzina et Sanfilippo, 2003; Luconi et Tintori, 2004). En effet, d'un côté, une partie des communautés à l'étranger est composée d'antifascistes qui ne veulent et ne peuvent pas rentrer; d'un autre côté, les autres sont prêts à tirer profit des initiatives du régime, sans pour autant être disposés à sacrifier leur réussite personnelle, qui ne semble pas pouvoir être atteinte dans les nouvelles destinations coloniales ou avec le retour en Italie (Capece, 2015; Ertola, 2017).

Les autorités fascistes déplorent la tendance à abandonner la campagne, mais toutes les initiatives visant à restructurer les grandes villes italiennes les transforment en lieux de migration. De plus, le régime fasciste planifie avec son allié nazi la reprise de l'émigration en Allemagne (Mantelli, 1992) et l'État se transforme en gestionnaire des départs. Le gouvernement italien découvre qu'il peut vendre les bras de ses citoyens en échange de fer, charbon, et/ou aides financiers. Cette politique constituera la base de la stratégie migratoire adoptée par le gouvernement démocrate-chrétien après la Seconde Guerre mondiale (Colucci, 2008).

Dans tous les cas, pendant le Ventennio fasciste, la principale destination des flux italiens reste l'Europe et les Amériques - même si les frontières sont fermées à une immigration massive - qui attirent les migrants plus que l'Afrique coloniale. Pendant la brève existence (1936-1941) de la soi-disant Africa orientale italiana, le départ vers l'ensemble des colonies italiennes dans la corne de l'Afrique ne constitue qu'entre un sixième et un septième des départs pour les Amériques (Ertola, 2017). 
Tableau 3 : Flux annuels d'émigrants italiens selon le continent de destination (1919-1945)

\begin{tabular}{lcccccc}
\hline Année & Europe & Afrique & Amériques & Asie & Océanie & Total \\
\hline 1919 & 134342 & 13092 & 105131 & 312 & 347 & 253224 \\
1920 & 198171 & 7303 & 408184 & 256 & 697 & 614611 \\
1921 & 79902 & 4840 & 114912 & 169 & 1468 & 201291 \\
1922 & 150555 & 4485 & 121139 & 865 & 4226 & 281270 \\
1923 & 199674 & 5496 & 183418 & 406 & 963 & 389957 \\
1924 & 232403 & 7044 & 120501 & 168 & 4498 & 364614 \\
1925 & 171630 & 6685 & 96435 & 149 & 5182 & 280081 \\
1926 & 134484 & 5474 & 117422 & 233 & 4783 & 262396 \\
1927 & 81801 & 4574 & 125463 & 196 & 6900 & 218934 \\
1928 & 62471 & 2943 & 73010 & 79 & 2353 & 140856 \\
1929 & 94342 & 5047 & 73766 & 202 & 1445 & 174802 \\
1930 & 155157 & 9568 & 69723 & 270 & 1720 & 236438 \\
1931 & 112322 & 12857 & 39693 & 280 & 708 & 165860 \\
1932 & 51666 & 6963 & 23578 & 244 & 897 & 83348 \\
1933 & 54626 & 6160 & 20766 & 273 & 1239 & 83064 \\
1934 & 36659 & 5683 & 23598 & 1188 & 1333 & 68461 \\
1935 & 26250 & 4394 & 24623 & 465 & 1676 & 57408 \\
1936 & 18098 & 3896 & 18248 & 286 & 1182 & 41710 \\
1937 & 25718 & 4191 & 27354 & 310 & 2372 & 59945 \\
1938 & 30570 & 3398 & 24645 & 209 & 2726 & 61548 \\
1939 & 11345 & 2086 & 14138 & 100 & 1820 & 29489 \\
1940 & 46968 & 528 & 3852 & 45 & 424 & 51817 \\
1941 & 8734 & 75 & & & & 8809 \\
1942 & 8216 & 30 & & & & 8246 \\
\hline & & & & & &
\end{tabular}

\section{Après la Seconde Guerre mondiale}

Après le conflit mondial, les flux migratoires vers les pays de l'Europe et vers I'Italie du Nord reprennent de manière désordonnée en raison de l'interaction instable entre la difficile situation économique interne et la demande étrangère de main-d'œuvre (De Clementi, 2010). L'Italie, à cause des ravages du conflit, doit être reconstruite, mais les autres nations manquent aussi de bras. Le développement de l'émigration clandestine, qui est une constante dans la diaspora italienne jusqu'à nos jours, est la conséquence des difficultés à résoudre ces contradictions (Rinauro, 2009); et de ce fait, le choix des destinations par les migrants reste très inconstant, même lorsque le gouvernement italien signe des accords concernant l'envoi de main-d'œuvre dans les autres pays européens (Colucci, 2008).

Dans les années 1946-1948, les pays d'Europe occidentale constituent la destination privilégiée de l'émigration italienne. Entre 1949 et 1950, les départs pour l'Europe diminuent et augmentent ceux pour l'Amérique latine et l'Australie; de 1951 à 1955, les destinations européennes, notamment France et Belgique, reprennent. Dans la deuxième moitié de la décennie, les flux vers la France et la Belgique diminuent alors que ceux vers la Suisse et l'Allemagne augmentent, mais il s'agit presque exclusivement de flux migratoires saisonniers. Par la suite, l'Allemagne prend progressivement de l'importance grâce à un accord signé entre I'Italie et la République fédérale allemande en 1955. Ce traité a eu des conséquences juridiques et législatives importantes puisqu'il constitue le point 
de départ de la gestion communautaire de la main-d'œuvre circulant en Europe occidentale (Colucci, 2015).

Les déplacements vers la Grande-Bretagne et les pays ne faisant pas partie de la communauté européenne sont moins consistants et réglementés (Colucci, 2009; Devoto, 2007; Pretelli, 2011 ; Bertagna, 2016). Dans la même période, nous assistons à des mouvements frontaliers entre la Ligurie, la France et la principauté de Monaco, ainsi qu'entre la Lombardie et la Suisse (Barcella et Colucci, 2016). En réalité, la migration pendulaire de part et $d$ 'autre $d$ 'une frontière ne relie pas seulement des villes et elle est souvent précédée par des déplacements d'une région à l'autre; de ce fait, les centres historiques des petites villes proches de la frontière française sont peuplés d'Italiens en provenance du Sud du pays.

Entre 1960 et 1980, les migrations internes à la Péninsule deviennent plus nombreuses que les migrations vers l'étranger, jusqu'à constituer la partie essentielle de la mobilité italienne (Colucci et Gallo, 2014-2016). Cette importance numérique génère un nouveau phénomène : les personnes qui migrent à l'étranger retournent le plus souvent en Italie, d'autant plus que, dans les années 1960, cette forme migratoire est souvent saisonnière ou programmée sur une courte période. Toutefois, même lorsque les migrants rentrent, ils ne s'installent plus au village d'origine, mais plutôt dans les villes du triangle industriel, de la province de Rome ou bien de la région de départ. C'est le cas des Abruzzesi qui à leur retour ne se rendent plus dans les villages des montagnes d'origine, mais préfèrent s'établir le long de la côte, surtout aux alentours de Pescara, une ville qui a connu une croissance rapide entre 1951 et 1971 (Sanfilippo, 2013).

À partir des années 1960, nous constatons une diminution des départs vers l'étranger et une augmentation massive des déplacements internes depuis le Sud vers le Centre et le Nord de I'Italie. Jusqu'en 1958, I'exode méridional se dirige vers les pays de l'Europe, l'Australie et les Amériques, tandis que les mouvements internes relient la campagne à la ville, la Vénétie (qui devient la région "championne de la mobilité interne") au triangle industriel, les Alpes orientales aux Alpes occidentales. Entre 1958 et 1963, les déplacements depuis le Sud vers le Nord du pays augmentent de façon exponentielle; au milieu des années 1960, ces flux sont marqués par une petite pause, mais ils reprennent en 1967 et changent définitivement le visage du pays, favorisant l'urbanisation de I'Italie septentrionale et centrale (Bonifazi, 2013 : chap. 4). Rome, qui profite de ces transformations, draine régulièrement les travailleurs provenant d'un vaste bassin, qui n'est pas seulement méridional, mais comprend aussi une bonne partie du Nord de l'Italie centrale. Ces migrations s'orientent aussi vers la région d'Émilie et notamment la ville de Bologne, ainsi que le Nord de la Toscane et la Ligurie du Ponant (la province d'Imperia, à la frontière avec la France). Entre temps, le nombre des migrants vers l'étranger, qui avait atteint une moyenne de 200 à 300000 départs par an pendant les années 1947-1968, descend à moins de 200000 entre 1969 et 1974, à 100000 entre 1975 et 1983, pour s'enliser, après 1986 , comme nous le verrons dans le paragraphe prochain. 
Tableau 4 : Flux annuels d'émigrants italiens selon le continent de destination (1946-1976)

\begin{tabular}{lcccccc}
\hline Année & Europe & Afrique & Amériques & Asie & Océanie & Total \\
\hline 1946 & 103077 & 82 & 7074 & 49 & 4 & 110286 \\
1947 & 192226 & 1439 & 60093 & 336 & 50 & 254144 \\
1948 & 193303 & 3567 & 108999 & 599 & 2047 & 308515 \\
1949 & 94959 & 4076 & 144122 & 373 & 10939 & 254469 \\
1950 & 54927 & 4740 & 126692 & 431 & 13516 & 200306 \\
1951 & 149206 & 8093 & 117462 & 662 & 17634 & 293057 \\
1952 & 144098 & 2774 & 103386 & 475 & 26802 & 277535 \\
1953 & 112069 & 3807 & 94874 & 1056 & 12865 & 224671 \\
1954 & 108557 & 3889 & 120813 & 704 & 16962 & 250925 \\
1955 & 149026 & 4258 & 114951 & 892 & 27699 & 296826 \\
1956 & 207631 & 4889 & 105946 & 696 & 25640 & 344802 \\
1957 & 236010 & 4156 & 84104 & 456 & 17007 & 341733 \\
1958 & 157800 & 2855 & 82298 & 122 & 12384 & 255459 \\
1959 & 192843 & 1445 & 59933 & 109 & 14160 & 268490 \\
1960 & 309876 & 1283 & 53042 & 78 & 19629 & 383908 \\
1961 & 329597 & 1022 & 40006 & 119 & 16379 & 387123 \\
1962 & 315795 & 706 & 34444 & 255 & 14411 & 365611 \\
1963 & 235134 & 589 & 30329 & 20 & 11539 & 277611 \\
1964 & 216498 & 1128 & 29788 & 178 & 10890 & 258482 \\
1965 & 232421 & 1390 & 38362 & 69 & 10401 & 282643 \\
1966 & 219353 & 2126 & 62365 & 102 & 12548 & 296494 \\
1967 & 166697 & 1927 & 46885 & 75 & 13680 & 229264 \\
1968 & 158462 & 2155 & 40563 & 28 & 14505 & 215713 \\
1969 & 139140 & 3690 & 29779 & 680 & 8910 & 182199 \\
1970 & 115114 & 3010 & 26563 & 627 & 6540 & 151854 \\
1971 & 133132 & 3209 & 24506 & 526 & 6348 & 167721 \\
1972 & 111908 & 2768 & 21930 & 653 & 4593 & 141852 \\
1973 & 98970 & 2438 & 18447 & 432 & 3515 & 123802 \\
1974 & 87105 & 3246 & 17142 & 754 & 3773 & 112020 \\
1975 & 72064 & 3461 & 13717 & 893 & 2531 & 92666 \\
1976 & 73031 & 4634 & 15266 & 1509 & 2807 & 97247 \\
\hline & & & & & &
\end{tabular}

\section{La fin des migrations italiennes?}

À partir des années 1970, les migrations internes et externes diminuent et le nombre des retours augmente, accompagné par la tendance déjà évoquée à s'installer dans une ville différente des villages d'origine (Corti, 2006 et 2013 : 25-29). En 1973, pour la première fois depuis plus d'un siècle, I'Italie enregistre un solde migratoire positif : le nombre de retours est légèrement supérieur à celui de départs. Même les mouvements frontaliers diminuent et, dans la décennie suivante, ils se réduisent de moitié (Barcella et Colucci, 2016). Toutefois, la mobilité au sein de la Péninsule ne s'arrête pas, tout comme la mobilité vers l'étranger dont la diminution sera effective seulement après 1985 (Bonifazi, 2013). En effet, elle continue à être alimentée par des flux réguliers de techniciens et d'ouvriers spécialisés qui partent tant vers les pays développés (Europe et Amérique du Nord) que vers les pays en développement (surtout Afrique et Amérique latine) auxquels s'ajoutent de nouveaux mouvements pour des causes politiques (Sanfilippo et Vignali, 2017). Après la Seconde Guerre mondiale, les fidèles de I'ancien régime fasciste partent; ils sont suivis par les personnes qui perdent dans la lutte pour 
les terres et par ceux qui voulaient contribuer à fonder le socialisme à l'Est de I'Europe centrale. Ensuite, tandis que des personnes appartenant à la bourgeoisie aisée craignant l'instabilité politique italienne, s'installent dans des pays jugés plus "tranquilles", comme le Canada et l'Australie, avec l'effondrement de leurs espoirs subversifs, les terroristes de droite se réfugient en Amérique latine et ceux de gauche en Amérique latine ou en France (Bertagna, 2006; ASEI, 2008).

La diminution des départs depuis I'Italie coexiste avec l'évolution des communautés italiennes à l'étranger. Comme dans les années 1920-1940, ces dernières se transforment et font preuve d'une grande vivacité avec la création de nouvelles organisations sociales et culturelles (Campani et Catani, 1985). Patronats, syndicats, partis politiques, groupes catholiques et laïques représentent tous encore une fois des points de repère importants pour les nouveaux arrivants, mais, à leur côté, s'organisent des associations nouvelles, souvent fondées sur des bases régionales ou provinciales (Colucci, 2001). Cette tendance est soutenue par la volonté de nombreuses régions qui tentent d'enrégimenter et d'utiliser leurs communautés à l'étranger (Martiniello, 1993).

Des liens économiques importants se nouent entre les anciennes régions de départs et les communautés émigrées : ce patronage politique devient important au XXle siècle avec l'adoption de la loi du 27 décembre 2001, no. 459, qui accorde le droit de vote aux Italiens émigrés (Tintori, 2012; Battiston et Mascitelli, 2012) ${ }^{7}$. Cette possibilité est donnée également aux descendants d'anciens émigrés italiens qui ont pu acquérir une double nationalité, parfois dans des conjonctures politiques difficiles, comme pour certains pays de l'Amérique du $\mathrm{Sud}^{8}$ et qui, grâce à la possession du passeport européen, ont pu émigrer du " Nouveau à l'Ancien Monde ». Entre temps, les émigrations italiennes en Europe et dans le monde reprennent, après avoir baissé entre 1977 et 2010, avec une forte contraction en 1987-1988, 1995-1997, 2002-2004 et 2007-2010.

Tableau 5 : Flux annuels d'émigrants italiens selon le continent de destination (1977-1990)

\begin{tabular}{lcccccc}
\hline Année & Europe & Afrique & Amériques & Asie & Océanie & Total \\
\hline 1977 & 65147 & 5915 & 12263 & 2299 & 2031 & 87655 \\
1978 & 61961 & 6533 & 11731 & 3224 & 2101 & 85550 \\
1979 & 67648 & 6346 & 10262 & 3089 & 1605 & 88950 \\
1980 & 64517 & 6120 & 9915 & 2678 & 1647 & 84877 \\
1981 & 68593 & 6292 & 9770 & 2668 & 1898 & 89221 \\
1982 & 75917 & 5599 & 11765 & 3155 & 1805 & 98241 \\
1983 & 64695 & 5794 & 9785 & 3387 & 1477 & 85138 \\
1984 & 60542 & 4736 & 8462 & 2296 & 1282 & 77318 \\
1985 & 50586 & 4345 & 8580 & 1983 & 1243 & 66737 \\
1986 & 44647 & 3383 & 7280 & 1499 & 1053 & 57862 \\
1987 & 26232 & 2617 & 7260 & 1112 & 1084 & 38305 \\
1988 & 25356 & 2432 & 6847 & 1145 & 880 & 36660 \\
1989 & 47760 & 2511 & 7295 & 1570 & 758 & 59894 \\
1990 & 36483 & 2368 & 7707 & 1255 & 1103 & 48916 \\
\hline
\end{tabular}

7 De fait, le droit à voter était déjà reconnu par la Constitution républicaine de 1947 (art. 48), mais les migrants devaient rentrer pour voter. La loi de 2001 a créé quatre circonscriptions électorales à l'étranger (Europe; Amérique méridionale; Amérique septentrionale; AfriqueAsie-Océanie), permettant aux migrants de voter sur place et d'y choisir leurs représentants. 8 Voir la circulaire du ministère de I'Intérieur, no. K.28.1.170 du 24 février 2003, au sujet des Argentins d'origine italienne. 


\section{Les nouvelles migrations du temps présent}

À partir des années 1990, les flux vers l'Europe occidentale et les États-Unis reprennent progressivement, tandis que les investissements économiques italiens dans l'Europe de l'Est favorisent la constitution des diasporas commerciales et/ou industrielles. Durant la dernière décennie du XXle siècle, des jeunes quittent I'Italie pour la Grande-Bretagne, I'Irlande, le Canada et les États-Unis; au début, ils partent comme touristes ou étudiants pour ensuite s'insérer sur le marché du travail, souvent d'une manière irrégulière (Caltabiano et Gianturco, 2005; Catania et al., 2010). Parallèlement, nous assistons aux premiers départs de chercheurs et professeurs universitaires, de stylistes et graphistes, de publicistes et réalisateurs, qui désirent travailler aux États-Unis et au Canada, ainsi qu'en France, en Allemagne et au Royaume-Uni (Brandi, 2006; Dubucs et al., 2017). Cette nouvelle mobilité, qui concerne surtout les personnes âgées de moins de quarante ans, est accompagnée par la reprise de l'émigration interne (Bonifazi et Heins, 2000 et 2009). La combinaison de ces différentes formes de migrations se relève complexe.

Les migrations internes des Italiens ont baissé jusqu'en 1991; après cette année, elles ont commencé à augmenter et pendant le quart de siècle suivant ont drainé la jeunesse dans le Mezzogiorno (Bonifazi, 2017). Avec le nouveau millénaire, un système à deux niveaux voit progressivement le jour : les migrants partent du Sud, parfois très jeunes, vers les régions du Centre et du Nord du pays, pour ensuite se déplacer vers d'autres pays européens. Dans les deux cas, au cours des années 2010, les pourcentages de départs sont significatifs; la migration interne a atteint de nouveaux pics, tandis que l'émigration d'Italiens vers l'étranger dépasse les chiffres de l'immigration. Après trente ans pendant lesquels les immigrants ont été majoritaires dans la Péninsule, I'Italie s'est retransformée en pays d'émigrants (Calvanese, 2000; Pugliese, 2006; Bonifazi, 2013; Corti, 2013). Après 2011, des migrants moins jeunes partent à côté des plus jeunes; il s'agit de personnes qui ont perdu leur travail à cause de la crise économique commencée en 2008 et de retraités, qui bénéficient d'un traitement économique qui ne leur permet pas de vivre en Italie à cause du coût de la vie (Sanfilippo et Vignali, 2017; Pugliese, 2017).

Les statistiques des années 2010 restituent des configurations migratoires très complexes. Pour saisir ces articulations, nous devons considérer les relations qui s'établissent entre mobilités interne et externe, ainsi que la coexistence dans les diasporas italiennes de personnes fortement qualifiés cherchant des postes de prestige, même pendant une courte durée, avec d'autres qui s'emploient dans n'importe quel type de travail. Ces migrants travaillent dans les cafés et les restaurants, les hôtels et les salons de coiffure, les magasins et les épiceries, la construction et l'entretien des bâtiments, alimentant ainsi les niches économiques traditionnelles de l'émigration italienne (Sanfilippo et Vignali, 2017). Dans ce cas, le travail précaire prédomine, mais reste mieux payé qu'en Italie. Bien que la presse italienne parle de la fuite de cerveaux, nous assistons à une fuite de main-d'œuvre, même intellectuelle, qui après la crise de 2008 ne trouve plus un travail dans la Péninsule et doit accepter un futur précaire à l'étranger, parfois alternant séjours à l'étranger et retours en Italie (FILEF, 2014; Caneva, 2016). 
Cette émigration est fluctuante et ne s'insère pas toujours dans les réseaux structurés des communautés d'origine italienne en Europe, aux Amériques et en Australie. Étant donné leur mobilité incessante, les personnes tendent plutôt à rester isolées. Par ailleurs, la recherche constante de travail pousse les nouveaux migrants à considérer les opportunités de partir vers des continents comme I'Asie ou des pays comme la Scandinavie, qui n'étaient pas des destinations habituelles. Les pays d'émigration traditionnelle ne sont pas pour autant abandonnés; nous assistons à une reprise de la mobilité vers l'Allemagne, le Royaume-Uni, la France, les États-Unis et l'Amérique latine (Gjergji, 2015). La complexité du phénomène est liée au fait que les familles partent, mais également des personnes seules et non plus seulement des hommes (Fondazione Migrantes, 2016 et 2017). II est vrai que nous devrions repenser le rapport homme/femme dans la composition des flux migratoires du XIXe et du $\mathrm{XXe}$ siècle et considérer le fait que déjà à cette époque des femmes partaient seules pour chercher du travail à l'étranger (Miranda, 2001; Miranda et Signorelli, 2011 ; Corti, 2013). Toutefois, la féminisation de la migration est beaucoup plus évidente dans la dernière phase migratoire; presque la moitié des personnes qui partent sont des femmes.

Tableau 6 : Flux annuels d'émigrants italiens selon le continent de destination (1991-2015)

\begin{tabular}{lcccccc}
\hline Année & Europe & Afrique & Asie & Amériques & Océanie & Total \\
\hline 1991 & 39313 & 1708 & 1147 & 8392 & 918 & 51478 \\
1992 & 37631 & 1576 & 2516 & 7668 & 835 & 50226 \\
1993 & 41163 & 1434 & 3331 & 8166 & 886 & 54980 \\
1994 & 47320 & 1637 & 1329 & 8368 & 748 & 59402 \\
1995 & 25697 & 1342 & 1147 & 6121 & 579 & 34886 \\
1996 & 28101 & 1650 & 1287 & 7396 & 583 & 39017 \\
1997 & 25920 & 2146 & 1575 & 8919 & 424 & 38984 \\
1998 & 25452 & 2055 & 1581 & 8493 & 567 & 38148 \\
1999 & 37700 & 3036 & 2316 & 12664 & 567 & 56283 \\
2000 & 31863 & 2643 & 1961 & 10526 & 487 & 47480 \\
2001 & 31388 & 2526 & 1935 & 10587 & 465 & 46901 \\
2002 & 21915 & 2576 & 2037 & 6997 & 262 & 33787 \\
2003 & 26564 & 2348 & 1520 & 8935 & 405 & 39772 \\
2004 & 28101 & 1581 & 1224 & 7641 & 533 & 39080 \\
2005 & 30243 & 1598 & 1374 & 8042 & 674 & 41931 \\
2006 & 32371 & 1604 & 1656 & 9743 & 872 & 46246 \\
2007 & 25310 & 1292 & 1645 & 7374 & 670 & 36291 \\
2008 & 27839 & 1358 & 1835 & 7774 & 730 & 39536 \\
2009 & 27586 & 1177 & 1936 & 7613 & 712 & 39024 \\
2010 & 27706 & 1381 & 2167 & 7553 & 734 & 39541 \\
2011 & 34332 & 1769 & 2907 & 10060 & 984 & 50052 \\
2012 & 47956 & 2452 & 3604 & 12544 & 1436 & 67992 \\
2013 & 58933 & 2418 & 4484 & 14603 & 1646 & 82084 \\
2014 & 64825 & 2510 & 4833 & 14741 & 1934 & 88843 \\
2015 & 76998 & 2761 & 5273 & 15041 & 2179 & 102252 \\
\hline & & 1393 &
\end{tabular}

Source : C. Perillo (Fondazione CSER, Rome), données ISTAT. 


\section{Conclusion}

En 2017, la comparaison des bases de données sur les migrations italiennes avec celles des autres pays européens suggère que l'incidence du nombre de départs est supérieure à celle qui est rapportée par les statistiques italiennes et qu'on pourrait multiplier les chiffres italiens par 2,5, voire 3, sinon plus (Cevoli et Ricci, 2017). Un exemple : entre 2011 et 2015, I'ISTAT (le bureau des statistiques italiennes) a comptabilisé 60700 départs vers l'Allemagne et le Statistisches Bundesamt $^{9}, 274285$. Si tel est le cas, nous serions en présence d'un phénomène dont la consistance rappelle celle de la grande migration qui a suivi la période après la Seconde Guerre mondiale (Pugliese, 2018). II est peut-être trop tôt pour évaluer cette hypothèse, mais il est évident que la nouvelle mobilité italienne vers l'étranger et à l'intérieur de la Péninsule est très élevée (Tirabassi et del Pra', 2014). De plus, I'immigration vers I'Italie est en décroissance à cause de la situation internationale (Strozza et De Santis, 2017) et plusieurs personnes des deuxièmes générations d'immigrants sont en train de chercher du travail à l'étranger (Fondazione Migrantes, 2016 et 2017). Nous ne pouvons pas évaluer tous les aspects de ce nouveau modèle migratoire italien. Les études sur les migrations qui ont suivi la Seconde Guerre mondiale ont, par exemple, souligné l'établissement de liens forts entre lieux de départ et d'arrivée (Miranda, 1997). Mais comment se reconfigurera la scène migratoire italienne avec les nouveaux départs auxquels nous assistons?

\section{Références bibliographiques}

Albera Dionigi e Corti Paola (Dirs.) (2000) La montagna mediterranea. Una fabbrica d'uomini? Mobilità e migrazioni in una prospettiva comparata (ss. $X V-X X)$, Cavallermaggiore, Gribaudo, $251 \mathrm{p}$.

Albonico Aldo e Rosoli Gianfausto (1994) Italia y América, Madrid, Mapfre, 449 p.

Archivio di Stato di Torino Consolati nazionali, Cadice, I, 1790-1835.

ASEI (2008) Per una storia politica dell'emigrazione, Archivio storico dell'emigrazione italiana, 4, $244 \mathrm{p}$.

Audenino Patrizia (2007) Emigrazione lombarda e modelli migratori dell'Italia settentrionale, in Ornella De Rosa e Donato Verrastro Dirs., Appunti di viaggio. L'emigrazione italiana tra attualità e memoria, Bologna, il Mulino, pp. 87-113.

Audenino Patrizia (1990) Un mestiere per partire. Tradizione migratoria, lavoro e comunità in una vallata alpina, Milano, Franco Angeli, 288 p.

Audenino Patrizia e Tirabassi Maddalena (2008) Migrazioni italiane: storia e storie dall'ancien régime a oggi, Milano, Bruno Mondadori, 213 p.

Baldoli Claudia (2004) Un fallimento del fascismo all'estero. La costruzione delle piccole Italie nella Germania nazista, Italia contemporanea, 235, pp. 221-238.

Baldoli Claudia (2003) Exporting Fascism: Italian Fascists and Britain's Italians in the 1930s, New York-Oxford, Berg, 288 p.

9 L'équivalent en Allemagne de l'Institut national de la statistique et des études économiques. 
Barcella Paolo e Colucci Michele (Dirs.) (2016) Frontalieri, Archivio storico dell'emigrazione italiana, 12, pp. 1-73.

Battiston Simone e Mascitelli Bruno (2012) Voto italiano all'estero. Riflessioni, esperienze e risultati di un'indagine in Australia, Firenze, Firenze University Press, $144 \mathrm{p}$.

Bertagna Federica (2016) Empresas, empresarios e inmigrantes italianos en la Argentina del primer peronismo (1946-1955), Revista de historia industrial, 62, p. 177-208.

Bertagna Federica (2006) La patria di riserva. L'emigrazione fascista in Argentina, Roma, Donzelli, 298 p.

Bertolotti Davide (1822) Peregrinazioni, I, Milano, Società tipografica dei Classici italiani, $142 \mathrm{p}$.

Bertolotti Davide (1820a) II Lago d'Orta. II natio della Riviera d'Orta in Buenos Ayres, II Raccoglitore, VII, Milano, Società tipografica dei Classici italiani, pp. 56-63.

Bertolotti Davide (1820b) Ancora il natio della Riviera d'Orta in America, II Raccoglitore, VII, Milano, Società tipografica dei Classici italiani, pp. 113-131.

Bevilacqua Piero, De Clementi Andreina e Franzina Emilio (Dirs.) (2001) Storia dell'emigrazione italiana, I, Partenze, Roma, Donzelli, 701 p.

Birindelli Anna Maria, Gesano Giuseppe e Sonnino Eugenio (1978) Lo spopolamento in Italia nel quadro dell'evoluzione migratoria e demografica (1871-1971), in Gianfausto Rosoli Dir., Un secolo di emigrazione italiana (1876-1976), Roma, CSER, pp. 189-250.

Blanc-Chaléard Marie-Claude, Bechelloni Antonio, Deschamps Bénédicte, Dreyfus Michel et Vial Éric (2007) Les Petites Italies dans le monde, Rennes, PUR, 444 p.

Bonifazi Corrado (Dir.) (2017) Migrazioni e integrazioni nell'Italia di oggi, Roma, CNR-IRPPS, $414 \mathrm{p}$.

Bonifazi Corrado (2013) L'Italia delle migrazioni, Bologna, il Mulino, 299 p.

Bonifazi Corrado e Heins Frank (2009) Ancora migranti: la nuova mobilità degli italiani, in Paola Corti e Matteo Sanfilippo Dirs., Storia d'Italia. Annali 24. Migrazioni, Torino, Einaudi, pp. 505-528.

Bonifazi Corrado and Heins Frank (2000) Long-term Trends of Internal Migration in Italy, International Journal of Population Geography, 6 (2), pp. 111-131.

Brandi Maria Carolina (2006) Le migrazioni delle alte professionalità tra mobilità internazionale e brain drain, Affari Sociali Internazionali, 34 (3), pp. 69-76.

Bujatti Anna (Dir.) (1992) Bertolotti Davide. Il filtro degli inchi, Palermo, Sellerio, $64 \mathrm{p}$.

Caltabiano Cristiano e Gianturco Giovanna (Dirs.) (2005) Giovani oltre confine. I discendenti e gli epigoni dell'emigrazione italiana nel mondo, Roma, Carocci, $428 \mathrm{p}$.

Calvanese Francesco (2000) L'Italia tra emigrazione e immigrazione, Roma, FILEF, $326 \mathrm{p}$. 
Campani Giovanna et Catani Maurizio (1985) Les réseaux associatifs italiens en France et les jeunes, Revue Européenne des Migrations Internationales, 1 (2), pp. $143-160$.

Caneva Elena (2016) Giovani italiani che emigrano: percorsi di vita inediti all'epoca della crisi economica globale, Mondi Migranti, 3, pp. 79-93.

Capece Francesca (2015) II rimpatrio degli italiani all'estero durante il fascismo: elementi e riflessioni a partire da un caso di studio italo-marsigliese, Archivio storico dell'emigrazione italiana, 11, pp. 71-79.

Cappelli Vittorio (2015) Rotte migratorie calabro-lucane (e cilentane) in America Latina. Percorsi artistico-culturali e sviluppo urbano in Brasile tra '800 e '900, Basiliskos, 2, pp. 57-73.

Castronovo Valerio (Dir.) (1986-1997) Biellesi nel mondo, Milano, Electa,7 vols.

Catania Danilo, Luconi Stefano e Zucca Gianfranco (2010) Guardando I'oceano da un grattacielo, Viterbo, Sette Città, 254 p.

Cazzola Franco (1983) Strutture agricole e crisi sociale nella Valle padana del secondo Ottocento, Annali dell'Istituto Alcide Cervi, 5, pp. 11-51.

Cevoli Marida e Ricci Rodolfo (2017) Le nuove migrazioni italiane, in Fondazione Di Vittorio, (Im)migrazione e sindacato, VIII Rapporto, Roma, Ediesse, chap. 13.

Colucci Michele (2015) Dall'Italia all'Europa: le migrazioni dopo il 1945, in Fabio Fabbri Dir., Storia del lavoro in Italia, II Novecento, II, 1945-2000, Roma, Castelvecchi, pp. 257-291.

Colucci Michele (2009) Emigrazione e ricostruzione. Italiani in Gran Bretagna dopo la seconda guerra mondiale, Foligno, Editoriale Umbra, 130 p.

Colucci Michele (2008) Lavoro in movimento. L'emigrazione italiana in Europa, 1945-57, Roma, Donzelli, 262 p.

Colucci Michele (2001) L'associazionismo di emigrazione nell'Italia repubblicana, in Piero Bevilacqua, Andreina De Clementi e Emilio Franzina Dirs., Storia dell'emigrazione italiana, I, Partenze, Roma, Donzelli, pp. 415-429.

Colucci Michele e Gallo Stefano (Dirs.) (2016) Fare spazio. Rapporto 2016 sulle migrazioni interne in Italia, Roma, Donzelli, 170 p.

Colucci Michele e Gallo Stefano (Dirs.) (2015) Tempo di cambiare. Rapporto 2015 sulle migrazioni interne in Italia, Roma, Donzelli, $172 \mathrm{p}$.

Colucci Michele e Gallo Stefano (Dirs.) (2014) L'arte di spostarsi. Rapporto 2014 sulle migrazioni interne in Italia, Roma, Donzelli, 172 p.

Corsini Carlo Alberto (1993) Le migrazioni interne e a media distanza in Italia: 1500-1900, in Commission Internationale de Démographie Historique, Actes de la 1re Conférence Européenne, vol. 3, Santiago de Compostela, CIDH, pp. 47-69.

Corsini Carlo Alberto (1969) Le migrazioni stagionali dei lavoratori nei Dipartimenti italiani del periodo napoleonico (1810-1812), in Saggi di Demografia storica, Firenze, Dipartimento Statistico Matematico, pp. 89-157.

Corti Paola (2013) Temi e problemi di storia delle migrazioni italiane, Viterbo, Sette Città, $132 \mathrm{p}$. 
Corti Paola (2006) Dal “ritorno" alle visits home: le tendenze di studio dell'ultimo trentennio, Studi Emigrazione, 164, pp. 835-856.

Corti Paola (2003) L'emigrazione italiana in Francia: un fenomeno di lunga durata, Altreitalie, 26, pp. 4-26.

Corti Paola (2000) L'emigrazione piemontese: un modello regionale?, Giornale di storia contemporanea, 3 (2), pp. 22-41.

Corti Paola (1990) Paesi d'emigranti. Comunità, mestieri, itinerari, identità collettive, Milano, Franco Angeli, 296 p.

Corti Paola e Sanfilippo Matteo (2012) L'Italia e le migrazioni, Roma-Bari, Laterza, $183 \mathrm{p}$.

Corti Paola e Sanfilippo Matteo (Dirs.) (2009) Storia d'Italia. Annali 24. Migrazioni, Torino, Einaudi, 803 p.

De Clementi Andreina (2010) II prezzo della ricostruzione. L'emigrazione italiana nel secondo dopoguerra, Roma-Bari, Laterza, 215 p.

De Clementi Andreina (1999) Di qua e di là dall'oceano. Emigrazione e mercati nel Meridione (1860-1930), Roma, Carocci, 144 p.

Degl'Innocenti Maurizio (Dir.) (1992) L'esilio nella storia del movimento operaio e l'emigrazione economica, Manduria, Lacaita, 300 p.

Devoto Fernando (2007) Storia degli italiani in Argentina, Roma, Donzelli, 504 p.

Dubucs Hadrien, Pfirsch Thomas, Recchi Ettore et Schmoll Camille (2017) Les migrations italiennes dans la France contemporaine. Les nouveaux visages d'une mobilité européenne, Hommes \& migrations, 1317-1318, pp. 59-67.

Ermacora Matteo (2013) L'altra strada. L'emigrazione interna dal Friuli (19191939), Meridiana, 75, pp. 85-108.

Ertola Emanuele (2017) In terra d'Africa. Gli italiani che colonizzarono l'impero, Roma-Bari, Laterza, 260 p.

Fauri Francesca (2015) Storia economica delle migrazioni italiane, Bologna, il Mulino, $240 \mathrm{p}$.

FILEF (2014) Le nuove generazioni nei nuovi spazi e nuovi tempi delle migrazioni, Roma, Ediesse, $176 \mathrm{p}$.

Fondazione Migrantes (2017) Rapporto italiani nel mondo 2017,Todi, Tau editrice, $510 \mathrm{p}$.

Fondazione Migrantes (2016) Rapporto italiani nel mondo 2016, Todi, Tau editrice, $502 \mathrm{p}$.

Franzina Emilio (2017a) Entre duas Pátrias. A Grande Guerra dos imigrantes ítalo-brasileiros 1914-1918, Belo Horizonte, Ramalhete, 380 p.

Franzina Emilio (Dir.) (2017b) Emigranti e profughi nel primo conflitto mondiale, Archivio storico dell'emigrazione italiana, 13, $175 \mathrm{p}$.

Franzina Emilio (2015) Militari italiani e la grande guerra, Zibaldone, Estudios italianos, $3(1)$, pp. 78-103. 
Franzina Emilio (2014) La terra ritrovata. Storiografia e memoria della prima immigrazione italiana in Brasile, Genova, Stefano Teramini Editore, 296 p.

Franzina Emilio (2001) Tirolesi italiani, cimbri veneti e modello di colonizzazione tedesco nella prima emigrazione agricola al Brasile (1875-1876), Memorie dell'Accademia Roveretana degli Agiati, 251, pp. 297-317.

Franzina Emilio (1995) Gli italiani al Nuovo mondo: l'emigrazione italiana in America, 1492-1942, Milano, Mondadori, 644 p.

Franzina Emilio (1982) La chiusura degli sbocchi emigratori, in Giovanni Cherubini, Franco Della Peruta, Ettore Lepore, Giorgio Mori, Mario Mazza, Giuliano Procacci e Rosario Villari Dirs, Storia della società italiana, XXI, La disgregazione dello Stato liberale, Milano, Teti, pp. 125-180.

Franzina Emilio (1976) La grande emigrazione. L'esodo dei rurali dal Veneto durante il secolo XIX, Venezia, Marsilio, 314 p.

Franzina Emilio e Sanfilippo Matteo (Dirs.) (2003) Il fascismo e gli emigrati. La parabola dei Fasci italiani all'estero (1920-1943), Roma-Bari, Laterza, 232 p.

Gallo Stefano (2015) II Commissariato per le migrazioni e la colonizzazione interna (1930-1940). Per una storia della politica migratoria del fascismo, Foligno, Editoriale Umbra, 222 p.

Gallo Stefano (2012) Senza attraversare le frontiere. Le migrazioni interne dall'Unità a oggi, Roma-Bari, Laterza, 215 p.

Gastaut Yvan et Kronenberger Stéphane (Dirs.) (2014) La Première Guerre mondiale et les migrations, Migrations Sociétés, 156, pp. 43-156.

Gioia Melchiorre (1804) Sul dipartimento del Lario. Discussione economica, Milano, Pirotta e Maspero, $332 \mathrm{p}$.

Gjergji Iside (Dir.) (2015) La nuova emigrazione italiana. Cause, mete e figure sociali, Venezia, Edizioni Ca' Foscari, 179 p.

Grossutti Javier (2009) Non fu la miseria, ma la paura della miseria. La colonia della Nuova Fagagna nel Chaco argentino (1877-1881), Udine, Forum, 144 p.

Isabella Maurizio (2014) Risorgimento in esilio. L'internazionale liberale e l'età delle rivoluzioni, Roma-Bari, Laterza, $382 \mathrm{p}$.

Lorenzetti Luigi e Merzario Raul (2005) // fuoco acceso. Famiglie e migrazioni alpine nell'Italia dell'età moderna, Rome, Donzelli, $194 \mathrm{p}$.

Luconi Stefano e Pretelli Matteo (2008) L'immigrazione negli Stati Uniti, Bologna, il Mulino, 214 p.

Luconi Stefano e Tintori Guido (2004) L'ombra lunga del fascio: canali di propaganda fascista per gli italiani d'America, Milano, M \& B Publishing, 154 p.

Mantelli Brunello (1992) "Camerati del lavoro". L'arruolamento di lavoratori italiani per il Terzo Reich nel periodo dell'Asse 1938-1943, Firenze, La Nuova Italia, $481 \mathrm{p}$.

Martiniello Marco (1993) L'associationnisme régional italien en Belgique : point final de l'immigritude?, dans Michel Dumoulin et Herman Van Der Wee Dirs., Hommes, cultures et capitaux dans les relations italo-belges aux XIXe et $X X e$ siècles, Rome et Bruxelles, Institut Historique Belge de Rome, pp. 23-39. 
Milza Pierre (Dir.) (1986) Les Italiens en France de 1914 à 1940, Rome, École française de Rome, $788 \mathrm{p}$.

Miranda Adelina (Dir.) (2001) Femmes italiennes en France. L'émigration féminine entre passé, présent et futur, Migrations Société, 78, 262 p.

Miranda Adelina (1997) Pendolari di ieri e pendolari di oggi. Storia di un paese di migranti, Torino, L'Harmattan-Italia, $144 \mathrm{p}$.

Miranda Adelina e Signorelli Amalia (2011) Pensare e ripensare le migrazioni, Palermo, Sellerio, 380 p.

Mourlane Stéphane et Païni Dominique (Dirs.) (2017) Ciao Italia! Un siècle d'immigration et de culture italiennes en France, Paris, La Martinière, 192 p.

Nani Michele (2016) Migrazioni bassopadane. Un secolo di mobilità residenziale nel Ferrarese (1861-1971), Palermo, NDF, 323 p.

Pizzorusso Giovanni (2009) Migrazioni di lavoro: la penisola italiana in età moderna, in Paola Corti e Matteo Sanfilippo Dirs., Storia d'Italia. Annali 24. Migrazioni, Torino, Einaudi, pp. 41-54.

Pizzorusso Giovanni (2007) Mobilità e flussi migratori prima dell'età moderna: una lunga introduzione, Archivio storico dell'emigrazione italiana, 3, pp. 205-222.

Pizzorusso Giovanni (2001) I movimenti migratori in Italia in antico regime, in Piero Bevilacqua, Andreina De Clementi e Emilio Franzina Dirs., Storia dell'emigrazione italiana, I, Partenze, Roma, Donzelli, pp. 3-16.

Ponte Giovanni (1967) Bertolotti, Davide, dans Dizionario Biografico degli Italiani, vol. 9, Roma, Istituto dell'Enciclopedia Italiana, [online]. URL: http://www. treccani.it/enciclopedia/davide-bertolotti_(Dizionario-Biografico)/

Porcella Marco (2001) Premesse dell'emigrazione di massa in età prestatistica, in Piero Bevilacqua, Andreina De Clementi e Emilio Franzina Dirs., Storia dell'emigrazione italiana, I, Partenze, Roma, Donzelli, pp. 17-44.

Prencipe Lorenzo e Sanfilippo Matteo (2009) Per una storia dell'emigrazione italiana: prospettiva nazionale e regionale, in Alessandro Nicosia e Lorenzo Prencipe Dirs., Museo Nazionale Emigrazione Italiana, Roma, Gangemi, pp. 44-141.

Pretelli Matteo (2011) L'emigrazione italiana negli Stati Uniti, Bologna, il Mulino, $213 p$.

Pretelli Matteo (2010) I/ fascismo e gli italiani all'estero, Bologna, Clueb, 160 p.

Prezioso Stéphanie (2010) Les Italiens en France au prisme de I'engagement volontaire : les raisons de l'enrôlement dans la Grande Guerre (1914-1915), Cahiers de la Méditerranée, 81, pp. 147-163.

Pugliese Enrico (2006) L'Italia tra migrazioni internazionali e migrazioni interne, Bologna, il Mulino, $195 \mathrm{p}$.

Pugliese Enrico (2018) Quelli che se ne vanno. La nuova emigrazione italiana, Bologna, il Mulino, $160 \mathrm{p}$.

Pugliese Enrico (2017) Giovani e anziani nella nuova emigrazione italiana, in Corrado Bonifazi dir., Migrazioni e integrazioni nell'Italia di oggi, Roma, CNR-IRPPS, pp. 45-59. 
Renda Francesco (1977) I fasci siciliani (1892-1894), Torino, Einaudi, 382 p.

Revel Jacques (Dir.) (1996) Jeux d'échelles. La micro-analyse à l'expérience, Paris, EHESS-Gallimard, 248 p.

Rinauro Sandro (2009) II cammino della speranza: l'emigrazione clandestina degli italiani nel secondo dopoguerra, Torino, Einaudi, 436 p.

Rosoli Gianfausto (1990) L'immaginario dell'America nell'emigrazione italiana di massa, Bollettino di Demografia Storica, 12, pp. 189-207.

Russo Saverio e Salvemini Biagio (2007) Ragion pastorale, ragion di stato. Spazi dell'allevamento e spazi dei poteri nell'Italia di età moderna, Roma, Viella, 224 p.

Salvetti Patrizia (1998) Emigrazione e grande guerra tra renitenza e rimpatri, in Alessandra Staderini, Luciano Zani e Francesca Magni Dirs., La grande guerra e il fronte interno. Studi in onore di George Mosse, Camerino, Università degli Studi, pp. 207-234.

Sanfilippo Matteo (2016) Le migrazioni interne di antico regime, in Ornella De Rosa e Danilo Verrastro Dirs., Studi di storia contemporanea. Società, istituzioni, territori, Limena (PD), Webster, pp. 229-249.

Sanfilippo Matteo (2013) L'emigrazione abruzzese, in Mario Zanganella Dir., L'Aquila e l'Abruzzo nella storia d'Italia. economia, società, dinamiche politiche, Roma, Edizioni Nuova Cultura/Fondazione Ugo Spirito e Renzo De Felice, pp. 15-53.

Sanfilippo Matteo (2009) Gli esuli di antico regime, in Paola Corti e Matteo Sanfilippo Dirs., Storia d'Italia. Annali 24. Migrazioni, Torino, Einaudi, pp. 142-160.

Sanfilippo Matteo (2008) L'emigrazione italiana nelle Americhe in età pre-unitaria, Annali della Fondazione Luigi Einaudi, 42, pp. 65-79.

Sanfilippo Matteo e Vignali Luigi Maria (Dirs.) (2017) La nuova emigrazione italiana, Studi Emigrazione, 207,

Sergi Pantaleone (2010) Stampa migrante: giornali della diaspora italiana e dell'immigrazione in Italia, Soveria Mannelli, Rubbettino, $214 \mathrm{p}$.

Sori Ercole (1979) L'emigrazione italiana dall'Unità alla seconda guerra mondiale, Bologna, il Mulino, $512 \mathrm{p}$.

Surdich Francesco (2005) La Liguria e Genova, territorio di emigrazione e porto degli emigranti: un ventennio di studi e di ricerche, in Luciano Gallinari Dir., Genova una porta del Mediterraneo, Cagliari, Consiglio nazionale delle ricerche, Istituto di storia dell'Europa mediterranea, pp. 952-1008.

Statistica Generale del Regno d'Italia (1884) Censimento degli italiani all'estero (31 dicembre 1881), Roma,Tipografia nell'Ospizio di San Michele, 106 p.

Statistica Generale del Regno d'Italia (1874) Censimento degli italiani all'estero (31 dicembre 1871), Roma, Stamperia Reale, 136 p.

SVIMEZ (2001-2016) Rapporto sull'economia del Mezzogiorno, Bologna, il Mulino, 16 vols.

Tintori Guido (Dir.) (2012) II voto degli altri: rappresentanza e scelte elettorali degli italiani all'estero, Torino, Rosenberg \& Sellier, 224 p. 
Tirabassi Maddalena e del Pra' Alvise (2014) La meglio Italia. Le mobilità italiane nel XXI secolo, Torino, Accademia University Press, $225 \mathrm{p}$.

Treves Anna (1976) Le migrazioni interne nell'Italia fascista, Torino, Einaudi, $203 \mathrm{p}$.

Vial Éric (2007) L'union populaire italienne 1937-1940: Une organisation de masse du Parti communiste italien en exil, Rome, École française de Rome, 461 p.

Viazzo Pier Paolo (2016) Internal and International Mobilities in the Alps, in Bruno Riccio Dir., From Internal to Transnational Mobilities, Bologna, I Libri di Emil/Odoya, pp. 137-153. 


\section{Matteo Sanfilippo}

\section{Les migrations italiennes : un aperçu statistique sur la longue durée}

Pour saisir la nature des flux migratoires enregistrés par les statistiques italiennes à partir des années 1870-1880, nous devons garder à l'esprit une longue histoire migratoire. Après la création du Royaume d'Italie (1861), les modalités migratoires à l'intérieur et à l'extérieur du pays ne changent pas. Comme depuis le Bas Moyen Âge, les mobilités italiennes externes interagissent pendant la deuxième moitié du $\mathrm{XIX}$ siècle avec les flux migratoires qui arrivent en Italie, se combinant entre eux ou bien alimentant la mobilité à l'intérieur de la Péninsule, notamment lorsqu'ils constituent une des étapes des migrations multiples.

\section{Italian Migration:}

\section{A statistical overview on the long term}

To understand the nature of the migration flows recorded by Italian statistics from the 1870s-1880s, we must keep in mind a long migration history. After the creation of the Kingdom of Italy (1861), migration patterns inside and outside the country do not change. As since the Late Middle Ages, external Italian mobility interacts during the second half of the 19th century with migratory flows arriving in Italy, combining with each other or fuelling mobility within the Peninsula, particularly when it constitutes one of the stages of multiple migration.

\section{Migración italiana: \\ una visión estadística a largo plazo}

Para comprender la naturaleza de los flujos migratorios registrados por las estadísticas italianas a partir de 1870-1880, debemos tener en cuenta una larga historia migratoria. Después de la creación del Reino de Italia (1861), los patrones de migración dentro y fuera del país no cambian. Como desde la Baja Edad Media, la movilidad externa italiana interactúa durante la segunda mitad del siglo XIX con los flujos migratorios que Ilegan a Italia, combinándose entre sí o alimentando la movilidad dentro de la Península, especialmente cuando constituye una de las etapas de la migración múltiple. 